\title{
APLICABILIDADE DAS TÉCNICAS DA TERAPIA COGNITIVO-COMPORTAMENTAL NO TRATAMENTO DE DEPRESSÃO E ANSIEDADE
}

\section{Applicability of cognitive-behavioral therapy techniques in depression and anxiety treatment}

\author{
Wildson Cardoso Assunção - FACIMAB/ Brasil \\ Jeann Bruno Ferreira da Silva - UFT/ UnirG / Brasil
}

\begin{abstract}
RESUMO: A depressão é considerada um problema de saúde pública a nível mundial. Estima-se que em 2020 ela será a segunda doença mais incapacitante em saúde. Dentre as características da depressão há, principalmente, alterações comportamentais disfuncionais. A ansiedade por sua vez pode ser caracterizada como devido a transtornos psiquiátricos ou como reações emocionais, envolvendo uma junção de sintomas físicos e alterações deficitárias no comportamento. A literatura mostra que há relação entre depressão e ansiedade. Objetivo: Este artigo de revisão objetiva descrever e refletir sobre as produções recentes que descreveram algumas das técnicas da Terapia Cognitivo-Comportamental - TCC utilizadas no tratamento de depressão e da ansiedade. Metodologia: Para construção da pesquisa foi escolhida a revisão narrativa da literatura, por ser uma metodologia apropriada para descrever e discutir o desenvolvimento de um assunto específico, de forma breve e a partir de uma perspectiva teórica ou contextual. Resultados e Discussão: Por utilizarmos de uma revisão narrativa da literatura, não foi possível descrever e refletir sobre a aplicabilidade das técnicas em casos específicos. Houve divergência quanto às definições de depressão e ansiedade. Em relação às técnicas de intervenção não houve tanta divergência na descrição e aplicabilidade dos procedimentos. Conclusão: A pesquisa constatou que a TCC é eficaz e dinâmica quanto aos processos e aplicabilidades das técnicas em depressão e ansiedade, mas que é necessário que o terapeuta reconheça a individualidade de cada paciente. Novas pesquisas são recomendadas para avaliar resultados de aplicação das técnicas em situações e contextos específicos.
\end{abstract}

Palavras-chave: Depressão e Ansiedade. Técnicas da Terapia CognitivoComportamental. Terapia Cognitivo-Comportamental.

ABSTRACT: Depression is considered a worldwide public health problem. It is estimated that by 2020 it will be the second most disabling health disease. Among the characteristics of depression there are mainly dysfunctional behavioral changes. Anxiety in turn can be characterized as due to psychiatric disorders or as emotional reactions involving a combination of physical symptoms and impaired behavioral changes. The literature shows that there is a relationship between depression and anxiety. Objective: This review article aims to describe and reflect on recent productions that have described some of the Cognitive Behavioral Therapy - CBT techniques used in the treatment of depression and anxiety. Methodology: For the construction of the research the narrative review of the literature was chosen because it is an appropriate methodology to describe and discuss the development of a specific subject, briefly and from a theoretical or contextual perspective. Results and Discussion: As we used a narrative literature review, it was not possible to describe and reflect on the applicability of the techniques in specific cases. There was disagreement 
as to the definitions of depression and anxiety. Regarding intervention techniques, there was not so much divergence in the description and applicability of the procedures. Conclusion: This research has found that CBT is effective and dynamic in the processes and applicability of techniques in depression and anxiety, but the therapist needs to recognize the individuality of each patient. New research is recommended to evaluate results of applying the techniques in specific situations and contexts.

Keywords: Depression and Anxiety. Techniques of Cognitive-Behavioral Therapy. Cognitive-Behavioral Therapy.

\section{INTRODUÇÃO}

A depressão é um problema de saúde que afeta pessoas no mundo todo (IONESCU, ROSENBSAUN e ALPERT, 2015) e estima-se que em 2020 ela será a segunda maior causa incapacitante em saúde (STOPA, et al., 2015). Abelha (2014) caracteriza a depressão como sendo a perda de interesse e prazer pelas coisas, seguido de uma baixa autoestima. A autora aponta que o pior desfecho em caso de não tratamento é o suicídio.

A depressão pode ser tratada através de terapias e/ou paralelo ao tratamento medicamentoso (DRIESSEN e HOLLON, 2010). A TCC trabalha com o tratamento de depressão utilizando diversos recursos estratégicos e técnicas, tem seus efeitos tão eficazes quanto o tratamento medicamentoso (CUIJPERS, et al., 2010/2013).

A ansiedade atinge grande parte da população no mundo todo e pode ser caracterizada como transtorno psiquiátrico ou como reação emocional disfuncional, envolve uma combinação de sintomas físicos, alterações no pensamento e comportamento (SILVA, 2012; BARCELLOS, et al., 2017). Em um contexto funcional, podemos dizer que a ansiedade é uma reação normal e necessária de uma pessoa (OBELAR, 2016).

Pesquisas mostram que a ansiedade é relacionada com a depressão, sendo apresentada na literatura como cada vez mais frequentes e em diversas populações. Minghelli, et al. (2010) Alali (2016) e Viana e Lourenço (2017) percebem que há relação entre transtornos de depressão e de ansiedade em diversas idades, bem como sintomas isolados dos mesmos em casos menos severos. 
Aplicabilidade das técnicas da terapia cognitivo-comportamental no tratamento de depressão e ansiedade

A Terapia Cognitivo-Comportamental - TCC é uma subárea da psicologia que estuda as relações entre cognição e comportamento, possui um aparato técnico-teórico direto, breve e específico e se caracterizada por ter sessões clínicas estruturadas e definidas, orientadas ao presente, trabalhando com demandas atuais (WRIGHT, BASCO e THASE, 2008; FARIA, 2011).

De acordo com Knapp e Beck (2008) as modificações cognitivas afetam e modificam a forma como nos comportamos. Em relação à terminologia terapia cognitivo-comportamental existe diferenças entre "terapia cognitiva" e "terapia comportamental", no entanto, atualmente essa separação ocorre apenas para fins literários (ASSUNÇÃO, 2018).

Dentre as possibilidades de intervenção da TCC, há o questionamento socrático (FARIA, 2011). O questionamento socrático teve sua origem com Sócrates (470 a.C 399 a.C), filósofo ateniense que utilizava uma técnica de "questionamento" denominada de maiêutica, cujo significado seria "dar a luz". Através desse recurso ele questionava seus oponentes em debates na busca pela "verdadeira verdade" (GOTTSCHALK, 2010).

A arteterapia também é uma ferramenta a ser considerada em transtornos depressivos, apesar de que seu estudo no contexto clínico é recente e pouco abordado, trata-se de uma abordagem terapêutica não verbal e tem sido uma alternativa nas psicoterapias verbais tradicionais (CIASCA, 2017). Ainda dentre as possibilidades Viana (2012) menciona o coping (enfrentamento) como uma estratégia onde de regulação das emoções e do sofrimento e a gestão do problema.

O manejo de ansiedade, por sua vez, possui suas próprias técnicas de intervenção, o treinamento em relaxamento, por exemplo, possui resultados positivos quando são aplicados devidamente e seguidos diariamente pelos pacientes (WILLHELM, ANDRETTA e UNGARETTI, 2015).

Em estudos citados por Ferreira (2015) é dito que a TCC utiliza técnicas de intervenção como uma variedade de treinos comportamentais, visualização dos acontecimentos, higiene do sono e a psicoeducação. Essas técnicas são aprendidas no contexto clínico e treinadas fora dele pelo paciente, durante momentos de crises ou desconfortos.

Mululo, et al. (2009) apontam que há eficácia nas técnicas da TCC em manejo de ansiedade em transtornos mentais, no entanto, os autores deixam claro que a TCC 
não se restringe às suas técnicas, mas que seriam um a ferramenta de investigação dos sintomas trazidos pelos pacientes.

Há possibilidades de uso da TCC em tratamentos grupais, conforme Heldt, et al. (2008) há resultados positivos e a viabilidade de tratar mais pessoas ao mesmo tempo. Essa modalidade é útil em ambientes locais como hospitais e ambientes que necessitem abranger mais pessoas em menos tempo.

Diante de todas essas possibilidades o objetivo deste estudo foi descrever e refletir, através de uma revisão narrativa da literatura e abrangendo as línguas Portuguesa e Inglesa, produzidas nos últimos 10 anos, as produções mais relevantes que descreveram técnicas da TCC utilizadas no tratamento da depressão e da ansiedade.

\section{METODOLOGIA}

Para construção dessa pesquisa foi escolhida a revisão narrativa da literatura. De acordo com Ănima (2014) a seleção do conteúdo de uma revisão narrativa é variável, qualitativa e ampla. Sallum, Garcia e Sanches (2012) e Assunção (2018) afirmam que essa metodologia é apropriada para descrever e discutir o desenvolvimento de um assunto específico, a partir de uma perspectiva teórica ou contextual.

Baseando-se na concepção de que a TCC utiliza como ferramenta de intervenção as técnicas que visam modificação de comportamento, se buscou responder "Quais são as técnicas usadas pela Terapia Cognitivo-Comportamental no tratamento de depressão e ansiedade?". O período delimitado para as buscas foi dos últimos 10 anos (2008-2018) para descrever e refletir sobre conteúdos mais atuais.

Foram utilizados os idiomas Português e Inglês para as buscas, que foram realizadas através das palavras chaves além de combinações com termos como cognição, crenças distorcidas e técnicas da TCC. As bases de dados utilizadas foram a Biblioteca Virtual em Saúde (BVS), incluindo Medline, Lilacs, Scielo, Google Acadêmico e artigos de periódicos que trouxessem conteúdos pertinentes à pesquisa.

Os critérios de inclusão adotados para seleção de artigos precisariam ser produzidos entre 2008 e 2018; precisariam ser publicados em periódicos científicos (para o caso de artigos fora das bases de dados citadas acima); precisariam publicados na língua Portuguesa ou Inglesa. Foram excluídos artigos incompletos, que não estavam

Educação, Psicologia e Interfaces, Volume 3, Número 1, p. 77-94, Janeiro/Abril, 2019.

ISSN: 2594-5343. DOI: https://doi.org/10.37444/issn-2594-5343.v3i1.113 
Aplicabilidade das técnicas da terapia cognitivo-comportamental no tratamento de depressão e ansiedade

hospedados em sites oficiais e que não descreviam técnicas em tratamento de depressão e ansiedade.

A partir da pesquisa geral na BVS, foram encontrados 29.236 artigos com o descritor "depressão e ansiedade". Após esta pesquisa utilizou-se como filtro o descritor “depressão e ansiedade", nos idiomas Português e Inglês, envolvendo todos os aspectos clínicos, países e tipos de estudo, foram encontrados 5.242 artigos.

Aplicando os mesmos critérios, foram encontrados 313 artigos relacionados a "técnicas da terapia cognitivo-comportamental depressão ansiedade" os resultados ainda abordavam aspectos amplos e distintos dos objetivos do presente trabalho. Foram filtrados ao todo 48 artigos que compuseram esse trabalho.

Por utilizarmos de uma revisão narrativa da literatura, esta pesquisa limitou-se em descrever e refletir sobre as técnicas de intervenção da TCC no tratamento da depressão e ansiedade para responder algumas questões sobre os métodos mais discutidos de forma breve.

\section{RESULTADOS}

\subsection{TCC NO TRATAMENTO DE DEPRESSÃO}

\subsection{Questionamento Socrático}

Knapp e Beck (2008) afirmam que apesar das críticas, as técnicas da TCC não são rígidas como pareciam e seria necessário um engajamento denominado de "empirismo colaborativo" entre terapeuta e paciente.

Para a TCC o questionamento socrático é de uso essencial no decorrer das sessões clínicas. Inicialmente o questionamento socrático surgiu de um conceito filosófico que usa a lógica e segue regras da dialética. Foi criado por Sócrates (470 a.C - 399 a.C) e denominado por ele de "maiêutica", essa forma de questionamento existe até os dias de hoje na reflexão filosófica sobre métodos de ensino (GOTTSCHAL, 2010).

De acordo com Santos e Medeiros (2017) no contexto clínico o questionamento socrático sugere ao indivíduo uma forma de revisar seus próprios pensamentos e crenças distorcidas a fim de modificá-los, através de um confronto em forma de questionário a si mesmo. 
Porém, ainda segundo os mesmos autores, o questionamento socrático deve ser usado para tomada de consciência do paciente e ao invés de debates ou confrontos relacionados com sua situação, recomenda-se que o terapeuta conduza o paciente para que ele por si só chegasse a conclusões saudáveis.

Wright, Basco e Thase (2008) mencionam que além do questionamento socrático há uma técnica semelhante, denominada de "descoberta guiada". Essas duas técnicas seriam iniciadas e mediadas pelo terapeuta e ajudariam o paciente a realizar um exame de evidências favoráveis ao seu pensamento e de evidências contrárias, tendo como resultado um novo horizonte no que tange possibilidades futuras (SANTOS, MEDEIROS, 2017).

\subsection{Registro de Pensamento Disfuncional (RPD)}

Knapp e Beck (2008) definem o RPD como sendo um aliado para rastrear os pensamentos que surgiram pela situação estimuladora e que produzem diversos comportamentos. Na ótica dos autores o RPD capacita os pacientes a encontrar, desmistificar e modificar significados disfuncionais para uma compreensão mais racional.

O RPD é um instrumento de fácil compreensão, consiste basicamente em uma folha de papel onde são anotados pelo próprio paciente a hora, a situação, os pensamentos e as emoções envolvidas em situações cotidianas que envolvam mal estar.

Por se tratar de processos que envolvem emoções e considerando que a depressão tem como característica a desorganização das mesmas Powell, et al. (2008) informam que no RPD há um campo para anotação de pensamentos alternativos sobre a situação, com intuito de gerar outras possibilidades de arranjos cognitivos frente as mesmas situações.

\subsection{Descatastrofização}

A palavra catástrofe, conforme o dicionário Livre da Língua Portuguesa (2011) é um substantivo representa um grande desastre. Nessa perspectiva Lawrence e Wiebe (2017) mencionam que uma catástrofe se situa no presente e seria orientada para um futuro de duas vias, um de incertezas e o outro de indeterminação, permanecendo em uma singularidade, não somente nas noções de tempo, mas também de espaço. 
Aplicabilidade das técnicas da terapia cognitivo-comportamental no tratamento de depressão e ansiedade

A catastrofização é a elevação dos acontecimentos a um nível disfuncional que leva o individuo a idealizar reações incomuns no seu organismo a partir de sintomas tidos como comuns como, por exemplo, relacionar uma simples tontura com um início de ataque cardíaco (MANFRO, et al., 2008).

Lawrence e Wiebe (2017) descrevem a catastrofização do pensamento, onde haveria uma oposição entre um senso de realidade objetivo e um senso distorcido e subjetivo. Em estudos mencionados pelas autoras é dito que o processo do discurso da catastrofização gera uma oposição dialética entre o discurso real e o discurso catastrofizado a partir de pressupostos intrasubjetivos e socialmente construídos e que há uma problematização dos eventos, que por sua vez ocorreria na tentativa de compreender o que está acontecendo.

A descatastrofização é um recurso terapêutico rico, que consiste em uma intervenção onde o terapeuta vai propor ao paciente um teste à realidade de seus pensamentos negativos/catastrofizados. Trata-se de um somatório de técnicas, dentre elas: As projeções de tempo e questionamentos sobre possibilidades e desfechos de eventos ocorrerem (CANALS, et al., 2009).

\subsection{Arteterpia}

Dentre as técnicas de intervenção mais recentes há a intervenção com a arteterapia. Coqueiro, et al. (2010) afirmam que a arteterapia no contexto da saúde vem ganhando espaço, especialmente no campo da saúde mental. Conforme os autores, a arteterapia possibilita aos usuários novas perspectivas frente às aversões, medos e angústias.

Para Freitas (2010) a arteterapia é um instrumento importante no processo de construção subjetiva e no desenvolvimento pessoal, pois agiria diretamente na manutenção de uma autoconsciência. Na perspectiva da Associação de Arteterapia do Estado de São Paulo o uso da arte é tido com finalidades terapêuticas há séculos, porém, sua estruturação de recursos dentro das terapias é ainda muito recente (AAESP, 2010, p. $8)$.

Para Coqueiro, et al. (2010) a arteterapia seria um dispositivo terapêutico que teria integrado a si saberes das diversas áreas do conhecimento, tornando-se transdisciplinar. Os mesmos autores observam que o uso da arteterapia pode ser realizado em diversas faixas etárias. Em outras palavras, desde um tratamento 
convencional a um mais complexo e independente das faixas etárias é possível utilizar esse recurso como ferramenta de intervenção. No Brasil, apesar de pouco repercutido a arteterapia tem seu espaço consolidado e seus estudos se iniciaram na cidade de Goiânia/GO (FREITAS, 2010).

Segundo o autor atualmente existem diversos centros de formação em arteterapia, com ênfase em suas bases epistemológicas e em sua compreensão prática. Tais possibilidades nas técnicas com arteterapia demonstram que seus efeitos podem ser orientados junto às práticas convencionais da TCC devido seus efeitos positivos na cognição/comportamento do indivíduo.

Morris (2014) usa o termo terapia de arte cognitivo-comportamental e afirma que o uso da arte é também útil na TCC para reduzir a ansiedade e reduzir ataques de pânico e agorafobia.

\subsection{Coping}

Para Aziz, Klein e Treur (2011) o coping explicaria o papel das emoções positivas e negativas na avaliação estratégica de enfrentamento emocional focada na situação. Ou seja, ao avaliar a situação e analisar a relação desta com as emoções, era possível promover uma mudança.

Viana (2012) define o coping como uma estratégia onde haveria possibilidade de regulação das emoções/sofrimento e a gestão do problema. Conforme a autora essa avaliação definiria as estratégias de enfrentamento a serem realizadas, inclusive através de um instrumento denominado de Inventário de Estratégias de Coping (IEC).

Ainda seguindo essa concepção, haveria também uma possibilidade de ressignificar um evento aversivo e definir novas metas para resolução dos problemas. Em outras palavras, o coping pode ser definido como habilidade para estratégias desenvolvidas para o enfrentamento de situações até então aversivas.

Aziz, Klein e Treur (2011) enfatizam que é preciso considerar as diferentes formas com que as pessoas se adaptam e desenvolve as habilidades de enfrentamento. Os autores ainda mencionam que a abordagem cognitiva ao enfrentamento parte de um pressuposto onde, através de um processo, o indivíduo potencia a avaliação da situação.

Salvador (2016) afirma que historicamente o coping foi estudado pela psicanálise e era relacionado com mecanismos de defesa do ego, posteriormente, na 
Aplicabilidade das técnicas da terapia cognitivo-comportamental no tratamento de depressão e ansiedade

década de 60, o coping passou a ser estudado por cognitivistas que possuíam uma visão a partir do determinismo cognitivo e situacional.

\section{TCC NO MANEJO DA ANSIEDADE}

\subsection{Relaxamento e Respiração}

O relaxamento é uma técnica utilizada para reduzir a ansiedade (FARIA, 2011) a partir de uma perspectiva onde o paciente se perceba como um agente que controla a situação, ao invés do contrário. A técnica envolve procedimentos que variam desde controle respiratório até a criação de imagens mentais que facilitem o andamento da terapia.

Willhelm, Andretta e Ungaretti (2015) mostram que as técnicas comportamentais relativas à ansiedade podem trabalhar ao mesmo tempo mente e corpo. Tais intervenções são possíveis realizando técnicas de relaxamento, respiração diafragmática, relaxamento passivo e progressivo. Conforme os autores o relaxamento é a técnica mais utilizada em TCC e possibilita uma nova postura por parte do paciente frente ao tratamento.

As técnicas de respiração são as mais conhecidas, até por outras áreas da saúde, sendo mais pesquisadas no que diz respeito à ansiedade (WILLHELM, ANDRETTA e UNGARETTI, 2015). As técnicas envolvendo respiração variam de acordo com o objetivo pretendido. No caso da técnica da respiração diafragmática, por exemplo, é exercitada a respiração correta através de orientações sobre a postura e movimento pulmonar.

Marques e Delfino (2016) definem o relaxamento como uma forma do organismo em retornar ao seu estado natural/anterior, também relacionam o uso do relaxamento em manejo de estresse.

Os autores citam que em conjunto das técnicas de relaxamento há o treino respiratório, que treina o uso do músculo do diafragma durante o ciclo respiratório de forma mais eficaz do que a que nos adaptamos ao longo da vida.

Johannes Heinrich Schultz (1884-1970) desenvolveu a conhecida técnica do relaxamento autógeno (FILHO, 2009). O autor conceitua a questão do relaxamento como sendo algo de natureza psicofisiológica e, por este motivo, diretamente interligada. O treinamento autógeno seguiria princípios como indução e controle; ciclos de exercícios mentais; estados sugestionados e autonomia. 
O método de relaxamento criado por Edmund Jacobson (1888-1983) conhecido como Relaxamento Progressivo de Jacobson consiste em concentrar e relaxar os músculos através do pensamento induzido, objetivando reduzir a tensão desnecessária (NALAWADE e NALAWADE, 2016). Os autores enfatizam de que mesmo que a ansiedade não seja eliminada através desse método, a pessoa estaria com mais autonomia frente à situação.

Dentre as formas de relaxamento estão também o relaxamento muscular progressivo (RMP) que promove contração e relaxamento em grupos musculares; Imaginar situações confortáveis/visualização, que como o nome sugere, significa induzir o paciente a imaginar-se em situações que provoquem relaxamento; Meditação, que objetiva um bem estar físico e mental através da aceitação de pensamentos para posterior obtenção de habilidade para desfazer-se dele (FARIA, 2011).

\subsection{Higiene do Sono}

A Higiene do Sono se refere à qualidade da manutenção do sono. O sono é fundamental importância na vida de uma pessoa, é tido normalmente como período usado para descansar e repor as energias que foram gastas durante o dia e tendo qualidade restauradora (SOARES, 2011).

A falta do sono causaria sintomas que variam de gravidade e intensidade desde fadiga, cansaço até a falta de concentração e atenção, além de outros prejuízos psicológicos. Vigeta, et al. (2013) afirmam que durante o sono ocorrem reações únicas e essenciais para o organismo.

A higiene do sono consiste em procedimentos, também educativos, que favoreçam qualidade ao sono, e que o torne reparador. Ferreira (2015) recomenda uma rotina pré-sono, cerca de meia hora para realização de atividades como: contar estórias lúdicas para crianças (no caso de aplicação em crianças); realização de oração; reflexão interna; leitura; e até mesmo um simples banho.

Conforme a autora, a higiene do sono é um método eficaz e que auxilia na redução da ansiedade bem como em casos de insônia. Pinto, et al. (2012) afirmam que os hábitos antes do sono influenciam diretamente na qualidade do sono, e que em relação aos sintomas de ansiedade, depressão e estresse se manifestam de forma 
Aplicabilidade das técnicas da terapia cognitivo-comportamental no tratamento de depressão e ansiedade

diferente quando os hábitos envolvem idas a festas que tenham bebidas alcoolicas, cigarro, drogas, dentre outras substâncias que interfere no organismo.

\subsection{Psicoeducação}

A psicoeducação é método que foi desenvolvido em meados da década de 80 pelo pesquisador C. M. Anderson. Manfro, et al. (2008) afirmam que seu uso interrompe as reações de ansiedade e pânico através de elementos componentes que fornecem justificativas racionais a serem seguidas e no contexto clínico deve ser realizada nas primeiras sessões, podendo ser repetida durante o tratamento.

Conforme os autores, a psicoeducação atua tanto nos pensamentos de manutenção do medo e da ansiedade quanto em comportamentos de fuga e evitação. Já Bhattacharjee, et al. (2011) definem psicoeducação como uma série de intervenções didáticas adequadas para informação destinada aos pacientes e/ou seus familiares em relação a sua condição ou características comportamentais, de seus sintomas ou doenças.

Elias (2014) concorda com essa definição quando menciona que a psicoeducação é uma intervenção orientada para ajudar as pessoas em tratamento e seus familiares além da redução do estresse familiar, suporte social e encorajamento.

Para Lemes e Neto (2017) a psicoeducação também possui o objetivo de instruir, além de reunir instrumentos psicológicos e pedagógicos, por este motivo o termo psicoeducação.

\section{DISCUSSÃO}

Os estudos apresentaram divergências quanto às definições de depressão e ansiedade. Em estudos citados por Coêlho e Tourinho (2008) o conceito patológico da ansiedade é tido como impreciso devido às divergências nos sistemas explicativos e que tais divergências aconteceriam por causa dos constantes usos de metáforas que dificultaria a compreensão.

Percebeu-se também que as divergências ocorrem dependendo do enfoque que os autores dão às abordagens que seguiram em suas pesquisas. Em relação às técnicas de intervenção não há tanta divergência quanto à descrição das aplicabilidades e procedimentos, contudo, percebeu-se que cada autor empregou seu modo explicativo. 
Acredita-se que essas divergências ocorram devido à prática clinica dos pesquisadores e aos resultados obtidos nessas pesquisas, que podem variar de acordo com uma ampla diversidade de fatores. As divergências quanto às técnicas não são tão amplas quanto às divergências na explicação dos sintomas.

Por se tratar de um pequeno recorte na literatura, não foi possível descrever e refletir sobre a aplicabilidade das técnicas da TCC em casos específicos de ansiedade e depressão. As definições de depressão e ansiedade na ótica das pesquisas que fizeram uso da TCC mostram um consenso entre suas definições e seus métodos de intervenção. O levantamento também mostrou eficiência no uso das técnicas da TCC no tratamento de depressão e ansiedade, se mostrando claras, reconhecidas e utilizadas em um contexto mundial.

\section{CONSIDERAÇÕES FINAIS}

Os sintomas de depressão e ansiedade tem se tornado cada vez mais frequentes e em diversas populações. Esse conjunto de características e sintomas físicos e psicológicos podem causar sérios prejuízos clínicos em curtos períodos de tempo, especialmente em caso de não tratamento.

Depressão e ansiedade são disfunções complexas, podendo se manifestar devido alguma experiência aversiva ou condição psicopatológica. Todas as pessoas estão de alguma forma sujeitas aos seus sintomas.

A literatura apresenta a TCC como eficaz e dinâmica quanto aos processos e metodologias de tratamento. Apesar da discussão aqui enfatizada ter se mostrado clara quanto ao uso das técnicas, recomendam-se novas pesquisas para avaliar a funcionalidade de cada técnica em quadros de depressão ou ansiedade específicos, de forma que haja subsídios para a aplicação de métodos de intervenção em casos raros ou incomuns.

Vale lembrar que para melhor uso do amplo repertório de metodologias da TCC é necessário uma formação bem elaborada de uma aliança terapêutica com o paciente. Dessa forma, recomenda-se que o terapeuta tenha uma formação segura e consolidada para que o uso dessas técnicas tenham resultados e efeitos satisfatórios no tratamento.

\section{REFERÊNCIAS BIBLIOGRÁFICAS}

Educação, Psicologia e Interfaces, Volume 3, Número 1, p. 77-94, Janeiro/Abril, 2019. 
Aplicabilidade das técnicas da terapia cognitivo-comportamental no tratamento de depressão e ansiedade

ABELHA, L. Depressão, uma questão de saúde pública. Cad. Saúde Colet., 2014, 22 (3): 223, 2014. Disponível em: <www.scielo.br/pdf/cadsc/v22n3/1414-462X-cadsc-2203-0223.pdf>. Acesso em: 28/08/2018.

ALALI, T. The relationship between anxiety, depression and hopelessness among nonclinical sample. PlumX Metrics. 2016. Disponível em: <https://www.europsyjournal.com/article/S0924-9338(16)00296-0/fulltext>. Acesso em: 01/09/2018.

ASSUNÇÃO, W. C. Aspectos conceituais de terminologias relativas às abordagens cognitivas e comportamentais. Revista Amazônia Science \& Health. 2018. Disponível em: <http://ojs.unirg.edu.br/index.php/2/article/view/2636/pdf >. Acesso em: 12/01/2019.

AZIZ, A. A.; KLEIN, M. C. A; TREUR, J. Simulating Cognitive Coping Strategies for Intelligent Support Agents. 2011. Disponível em: <https://www.cs.vu.nl/ wai/Papers/CogSci10coping.pdf>. Acesso em 02/09/2018.

BARCELLOS, M. T.; et al. TeleConduta: Ansiedade. TelessaúdeRS - UFRGS. 2017. Disponível em:

<https://www.ufrgs.br/telessauders/documentos/telecondutas/Telecondutas_Ansiedade_ 20170331.pdf>. Acesso em: 28/08/2018.

BHATTACHARJEE, D.; et al. Psychoeducation: A Measure to Strengthen Psychiatric Treatment. Delhi Psychiatry Journal. 2011; 14:(1). Disponível em: <medind.nic.in/daa/t11/i1/daat11i1p33.pdf>. Acesso em: 02/09/2018.

CANALS, A. A.; et al. Terapia cognitivo-comportamental nos transtornos alimentares: uma abordagem familiar para intervenção em crise. Revista de Psicologia da IMED, vol.1, n.1, p. 56-71, 2009. Disponível em: <https://dialnet.unirioja.es/descarga/articulo/5154992.pdf>. Acesso em: 01/09/2018.

CIASCA, E. C. Arteterapia e depressão: Efeitos da arteterapia como terapia complementar no tratamento da depressão em idosos. Mestrado em Ciências Universidade de São Paulo. 2017. Disponível em: $<$ http://www.teses.usp.br/teses/disponiveis/5/5160/tde-06062017082522/publico/ElianaCeciliaCiascaVersaoCorrigida.pdf>. Acesso em: 28/08/2018.

COÊLHO, N. L.; TOURINHO, E. Z. O Conceito de Ansiedade na Análise do Comportamento. Psicologia: Reflexão e Crítica, 21(2), 171-178. 2008. Disponível em: <www.scielo.br/pdf/prc/v21n2/a02v21n2.pdf>. Acesso em: 28/08/2018.

COOKE, H. Progressive Muscle Relaxation. CAM-Cancer Consortium. 2015. Disponível em: <https://www.healthline.com/health/what-is-jacobson-relaxationtechnique >. Acesso em: 02/08/2018.

COQUEIRO, N. F.; et al.. Arteterapia como dispositivo terapêutico em saúde mental. Acta Paul Enferm 2010;23(6):859-62. Disponível em: <http://www.scielo.br/pdf/ape/v23n6/22.pdf>. Acesso em: 10/09/2016.

CUIJPERS, P.; et al. A Meta-Analysis of Cognitive-Behavioural Therapy for Adult Depression, Alone and in Comparison With Other Treatments. CanJPsychiatry. 
2013;58(7):376-38. Disponível em:

<journals.sagepub.com/doi/pdf/10.1177/070674371305800702>. Acesso em:

01/09/2018.

CUIJPERS, P.; et al. Efficacy of cognitive-behavioural therapy and other psychological treatments for adult depression: meta-analytic study of publication bias. The British

Journal of Psychiatry (2010) 196, 173-178. Disponível em:

<https://www.cambridge.org/core/services/aop-cambridge-

core/content/view/585841C1FAC63E0AAC140BA1557AEACA/S0007125000251805a

.pdf/efficacy_of_cognitivebehavioural_therapy_and_other_psychological_treatments_fo

r_adult_depression_metaanalytic_study_of_publication_bias.pdf $>$. Acesso em:

01/09/2018.

Dicionário livre da língua portuguesa. / Manoel M. Santiago-Almeida. — São Paulo: Hedra, 2011. 720 pp.

DRIESSEN, E.; HOLLON, S. D. Cognitive Behavioral Therapy for Mood Disorders: Efficacy, Psychiatr Clin North Am. 2010 Sep; 33(3): 537-555. Disponível em: <https://www.ncbi.nlm.nih.gov/pmc/articles/PMC2933381/>. Acesso em: 01/09/2018.

ELIAS, M. T. M. N. C. As Técnicas de Relaxamento como Instrumento Terapêutico de Enfermagem em Pessoas com Sofrimento Mental. Mestrado em Enfermagem - Escola Superior de Enfermagem de Lisboa. 2014. Disponível em:

<https://comum.rcaap.pt/bitstream/10400.26/16277/1/Relatório\%20Teresa\%20\%280505-2014\%29\%2016h23.pdf>. Acesso em: 03/09/2018.

FARIA, K. F. Uma revisão bibliográfica de técnicas cognitivo-comportamentais utilizadas nos transtornos de ansiedade generalizada, transtorno de pânico e fobia social. Monografia. 2011. Disponível em:

<https://lume.ufrgs.br/bitstream/handle/10183/40110/000826634.pdf>. Acesso em: 28/08/2018.

FERREIRA, R. E. R. F. Intervenção Comportamental para Problemas de Sono na Infância. Doutorado. Disponível em:

<www.teses.usp.br/teses/disponiveis/47/47133/tde-07072015.../ferreira_corrigida.pdf>. Acesso em: 08/09/2018.

FERREIRA; L. A. Psicoeducação como estratégia de higiene do sono em uma unidade básica de saúde. TCC. 2015. Disponível em:

<https://cepein.femanet.com.br/BDigital/arqTccs/1111370348.pdf>. Acesso em: 28/08/2018.

FILHO, P. G. S. Encontro paranaense, congresso Brasileiro de psicoterapias corporais, 2009. Disponível em: <www.centroreichiano.com.br/artigos/Anais2009/SOUSA-FILHO-Paulo-Gomes-Introducao-aos-metodos.pdf>. Acesso em: 02/08/2018.

FLÔRES, O. C.; GABRIEL, R. Da relação pensamento e linguagem ao estudo interdisciplinar da mente. Linguagem em (Dis)curso, Tubarão, SC, v. 12, n. 1, p. 155- 
Aplicabilidade das técnicas da terapia cognitivo-comportamental no tratamento de depressão e ansiedade

178, jan./abr. 2012. Disponível em: <www.scielo.br/pdf/ld/v12n1/v12n1a07.pdf>. Acesso em: 28/08/2018.

GOTTSCHALK, C. M. C. O papel do método no ensino: da maiêutica socrática à terapia wittgensteiniana. Educ. Tem. Dig., Campinas, v.12, n.1, p.64-81, jul./dez. 2010. Disponível em: <https://dialnet.unirioja.es/descarga/articulo/4856510.pdf>. Acesso em: 28/08/2018.

GRUPO ĂNIMA EDUCAÇÃO. Manual Revisão Bibliográfica Sistemática Integrativa: A pesquisa baseada em evidências. Grupo Ănima Educação, 2014. Disponível em: <disciplinas.nucleoead.com.br/pdf/anima_tcc/gerais/manuais/manual_revisao.pdf>. Acesso em: 09/09/2018.

HELDT, E. Utilização das técnicas da terapia cognitivo-compotamental em grupo para pacientes com sintomas residuais do transtorno de pânico: seguimento de 2 anos. Rev. HCPA, 2008;28(1). Disponível em:

<https://www.lume.ufrgs.br/bitstream/handle/10183/28907/000652269.pdf?sequence=1 >. Acesso em: 13/09/2018.

IONESCU, D. F.; ROSENBAUM, J. F.; ALPERT, J. E. Pharmacological approaches to the challenge of treatment-resistant depression. Dialogues Clin Neurosci. 2015; 17(2): 111-126. Disponível em: <https://www.ncbi.nlm.nih.gov/pmc/articles/PMC4518696/>. Acesso em: 01/09/2018.

KING, A. L. S.; VALENCA, A. M.; NARDI, A. E. Hiperventilação: A terapia cognitivo-comportamental e a técnica dos exercícios de indução dos sintomas no transtorno de pânico. Rev Port Pneumol., v. 14, n. 2, p. 303-308. 2008 . Disponível em: <http://www.scielo.mec.pt/scielo.php?script=sci_arttext\&pid=S087321592008000200012\&lng=pt\&nrm=iso $>$. Acesso em: 04//04/2018.

KNAPP, P.; BECK, A. T. Fundamentos, modelos conceituais, aplicações e pesquisa da terapia cognitiva. Rev Bras Psiquiatr. 2008;30(Supl II):S54-64. Disponível em: <www.scielo.br/pdf/rbp/v30s2/a02v30s2.pdf>. Acesso em: 28/08/2018.

LAWRENCE. J. L.; WEIBE, S. M. W. Biopolitical disaster. London; New York: Routledge, Taylor \& Francis Group, 2018.

LEMES, C. B.; NETO, J. O. Aplicações da psicoeducação no contexto da saúde. Temas psicol. vol.25 no.1. 2017. Disponível em:

<http://pepsic.bvsalud.org/pdf/tp/v25n1/v25n1a02.pdf>. Acesso em: 02/09/2018.

MANFRO, G. G.; et al. Cognitive-behavioral therapy in panic disorder. Rev. Bras.

Psiquiatr. vol.30 suppl. 2. 2008. Disponível em:

<http://www.scielo.br/pdf/rbp/v30s2/en_a05v30s2.pdf>. Acesso em: 12/08/2018.

MARQUES, E. L. L.; DELFINO, T. E. Contribuições das técnicas de respiração, relaxamento e mindfulness no manejo do estresse ocupacional. Monografia. 2016.

Disponível em: <www.psicologia.pt/artigos/textos/A0965.pdf>. Acesso em:

02/08/2018. 
MINGHELLI, B.; et al. Estudo qualitativo sobre a depressão e a ansiedade social na adolescência: uma revisão bibliográfica. Psicologia.pt. 2017. Disponível em: <www.psicologia.pt/artigos/textos/A1084.pdf>. Acesso em: 01/09/2018.

MINGHELLI, B.; et al. Comparação dos níveis de ansiedade e depressão entre idosos ativos e sedentários. Rev Psiq Clín. 2013;40(2):71-6. Disponível em: <www.scielo.br/pdf/rpc/v40n2/v40n2a04.pdf >. Acesso em: 01/09/2018.

MORRIS, F. J. Should art be integrated into cognitive behavioral therapy for anxiety disorders?. The Arts in Psychotherapy, v. 41, Issue 4. 343-352. 2014. Disponível em: $<$ https://www.sciencedirect.com/science/article/abs/pii/S0197455614000720>. Acesso em: 08/08/2018.

NALAWADE, T. C.; NIKHADE, N. S. Effectiveness of Jacobson Progressive Muscle Relaxation Technique on Depressive Symptoms and Quality of Life Enjoyment and Satisfaction in Community Dwelling Older Adults. Indian Journal of Basic and Applied Medical Research; 2016: Vol.-5, Issue- 4, P. 448-452. Disponível em: <ijbamr.com/pdf/September\%202016\%20448-452.pdf.pdf>. Acesso em: 02/08/2018.

OBELAR, R. M. Avaliação psicológica nos transtornos de ansiedade: estudos brasileiros. Monografia. 2016. Disponível em:

<https://www.lume.ufrgs.br/bitstream/handle/10183/147064/000998195.pdf?sequence= 1>. Acesso em: 02/08/2018.

PINTO, T. R.; et al. Hábitos de sono e ansiedade, depressão e estresse: Que relação? Actas do $12^{\circ}$ colóquio de psicologia e educação. Disponível em: <repositorio.ispa.pt/bitstream/10400.12/1616/1/CIPE\%202012\%20990-1006.pdf>. Acesso em: 03/09/2018.

POWELL, V. B. Terapia cognitivo-comportamental da depressão. Rev. Bras. Psiquiatr. vol.30 suppl.2 São Paulo Oct. 2008. Disponível em: <http://www.scielo.br/pdf/rbp/v30s2/en_a04v30s2.pdf>. Acesso em: 01/09/2018.

SALLUM, A. M. C.; GARCIA, D. M.; SANCHES, M. Dor aguda e crônica: revisão narrativa da literatura. Acta Paul Enferm. 2012;25(Número Especial 1):150-4.. Disponível em: <www.scielo.br/pdf/ape/v25nspe1/pt_23.pdf>. Acesso em: 28/08/2018.

SALVADOR, I. F. Avaliação da percepção do diagnóstico e dos processos de coping de pacientes leucêmicos. Mestrado em Psicologia - PUC Goiás. 2016. Disponível em: <tede2.pucgoias.edu.br:8080/bitstream/tede/1910/1/IZADORA\%20DE\%20FREITAS\% 20SALVADOR.pdf>. Acesso em: 02/08/2018.

SANTOS, C. E. M; MEDEIROS, F. A. A relevância da técnica de questionamento socrático na prática Cognitivo-Comportamental. Arch Health Invest (2017) 6(5):204208. Disponível em:

<archhealthinvestigation.com.br/ArcHI/article/download/1940/pdf>. Acesso em: 28/08/2018. 
Aplicabilidade das técnicas da terapia cognitivo-comportamental no tratamento de depressão e ansiedade

SILVA, L. F. V. A ansiedade e seu enfrentamento. Psico-USF, v. 17, n. 1, p. 165-166, jan./abr. 2012. Disponível em: <www.scielo.br/pdf/pusf/v17n1/a18v17n1.pdf>. Acesso em: 28/08/2018.

SOARES, M. J. R. C. Influência da qualidade do sono na performance dos atletas de alta competição. Mestrado em Ciências Biomédicas - Universidade do Porto. 2011. Disponível em: <https://repositorioaberto.up.pt/bitstream/10216/63628/2/tese\%20final.pdf>. Acesso em: 03/09/2018.

STOPA, S. R..; et al. Prevalência do autorrelato de depressão no Brasil: resultados da Pesquisa Nacional de Saúde, 2013. Rev Bras Epidemiol, 2015; 18 SUPPL 2: 170-180. Disponível em: <https://www.scielosp.org/pdf/rbepid/2015.v18suppl2/170-180/pt>. Acesso em: 28/08/2018.

VIANA, A. C. W. Terapia cognitivo-comportamental em grupo para transtorno de pânico: Avaliação de efeito do protocolo padrão e do acrescimo de sessões de reforço com técnicas cognitivas nas estratégias de enfrentamento (Coping). Mestrado em Ciências Médicas - Universidade Federal do Rio Grande do Sul. 2012. Disponível em: <https://www.lume.ufrgs.br/handle/10183/61733>. Acesso em: 02/08/2018.

VIANA, R. S.; LOURENÇO, L. M. Estudo qualitativo sobre a depressão e a ansiedade social na adolescência: uma revisão bibliográfica. Disponível em: <www.psicologia.pt/artigos/textos/A1084.pdf>. Acesso em: 09/09/2018.

VIGETA, S. M. G.; et al. O conhecimento da higiene do sono na menopausa. Rev APS. $2013 \mathrm{abr} / \mathrm{jun} ;$ 16(2): 122-128. Disponível em: <https://www.researchgate.net/publication/257991975_O_CONHECIMENTO_DA_HI GIENE_DO_SONO_NA_MENOPAUSA>. Acesso em: 03/09/2018.

WILLHELM, A. R.; ANDRETTA, I.; UNGARETTI, M. S. Importância das técnicas de relaxamento na terapia cognitiva para ansiedade. Contextos Clínicos, 8(1):79-86, janeiro-junho 2015. Disponível em: <pepsic.bvsalud.org/pdf/cclin/v8n1/v8n1a09.pdf>. Acesso em: 28/08/2018.

\section{Credenciais dos autores}

ASSUNÇÃO, Wildson Cardoso. Psicólogo Especialista em Terapia CognitivoComportamental pela Faculdade de Ciências Sociais Aplicadas de Marabá - FACIMAB, Brasil. Psicólogo Escolar e da Saúde na APAE de Porto Nacional/TO. E-mail: wildson.se@outlook.com

DA SILVA, Jeann Bruno Ferreira. Psicólogo, Doutorando em Desenvolvimento Regional pela Universidade Federal do Tocantins - UFT, Brasil. Professor na Universidade de Gurupi - UnirG. E-mail: jbpsicologia@live.com 
Endereço para correspondência: Wildson Cardoso Assunção. Rua Piracicaba Qd. 08, Lt. 06, n. 46, Setor Paulista, CEP: 77410-610. Gurupi/TO. E-mail: wildson.se@outlook.com

Como citar este artigo (Formato ABNT): ASSUNÇÃO, Wildson Cardoso; DA SILVA, Jeann Bruno Ferreira. Aplicabilidade das técnicas da terapia cognitivocomportamental no tratamento de depressão e ansiedade. Educação, Psicologia e Interfaces, v. 3, n.1, p. 77-94, 20119. DOI: https://doi.org/10.37444/issn-25945343.v3i1.113

Recebido: $14 / 08 / 2018$.

Aceito: 20/01/2019. 